\title{
Multipurpose modular experimental station for the DiProl beamline of Fermi@Elettra free electron laser
}

\author{
Emanuele Pedersoli, ${ }^{1}$ Flavio Capotondi, ${ }^{1}$ Daniele Cocco, ${ }^{1}$ Marco Zangrando, ${ }^{1,2}$ \\ Burkhard Kaulich, ${ }^{1}$ Ralf H. Menk, ${ }^{1}$ Andrea Locatelli, ${ }^{1}$ Tevfik O. Mentes, ${ }^{1}$ Carlo Spezzani, ${ }^{1}$ \\ Gilio Sandrin, ${ }^{1}$ Daniel M. Bacescu, ${ }^{1, a)}$ Maya Kiskinova, ${ }^{1, b)}$ Saša Bajt, ${ }^{3}$ \\ Miriam Barthelmess, ${ }^{3}$ Anton Barty, ${ }^{4}$ Joachim Schulz, ${ }^{4}$ Lars Gumprecht, ${ }^{4}$ \\ Henry N. Chapman, ${ }^{4,5}$ A. J. Nelson, ${ }^{6}$ Matthias Frank, ${ }^{6}$ Michael J. Pivovaroff, ${ }^{6}$ \\ Bruce W. Woods, ${ }^{6}$ Michael J. Bogan, ${ }^{7}$ and Janos Hajdu ${ }^{8}$ \\ ${ }^{1}$ Fermi, Elettra Sincrotrone Trieste, SS 14 - km 163.5, 34149 Basovizza, Trieste, Italy \\ ${ }^{2}$ IOM CNR, Laboratorio TASC, SS 14 - km 163.5, 34149 Basovizza, Trieste, Italy \\ ${ }^{3}$ Photon Science, DESY, Notkestrasse 85, 22607 Hamburg, Germany \\ ${ }^{4}$ Centre for Free-Electron Laser Science, DESY, Notkestrasse 85, 22607 Hamburg, Germany \\ ${ }^{5}$ University of Hamburg, Notkestrasse 85, 22607 Hamburg, Germany \\ ${ }^{6}$ Physical and Life Sciences, LLNL, 7000 East Avenue, Livermore, California 94550, USA \\ ${ }^{7}$ PULSE Institute, SLAC National Accelerator Laboratory, 2575 Sand Hill Rd, Menlo Park, \\ California 94025, USA \\ ${ }^{8}$ Laboratory of Molecular Biophysics, Uppsala University, Husargatan 3, Box 596, \\ 75124 Uppsala, Sweden
}

(Received 2 February 2011; accepted 28 March 2011; published online 28 April 2011)

\begin{abstract}
We present a compact modular apparatus with a flexible design that will be operated at the DiProI beamline of the Fermi@Elettra free electron laser (FEL) for performing static and time-resolved coherent diffraction imaging experiments, taking advantage of the full coherence and variable polarization of the short seeded FEL pulses. The apparatus has been assembled and the potential of the experimental setup is demonstrated by commissioning tests with coherent synchrotron radiation. This multipurpose experimental station will be open to general users after installation at the Fermi@Elettra free electron laser in 2011. ( ) 2011 American Institute of Physics. [doi:10.1063/1.3582155]
\end{abstract}

\section{INTRODUCTION}

\section{A. Coherent diffraction imaging (CDI) with free electron lasers (FELs)}

The advent of new generation free electron laser (FEL) sources, providing ultrashort pulses of high transverse coherence and peak brightness, has opened extraordinary opportunities for lensless coherent diffraction imaging (CDI) with regard to achievable spatial and temporal resolution. ${ }^{1,2}$ The pioneering results obtained at the first operating FEL facilities, FLASH in Hamburg and LCLS in Stanford, have clearly demonstrated that CDI is going to be a key technique for sampling building blocks of matter down to near-atomic resolution, by acquiring diffraction patterns, with sufficient intensity for a single-shot mode, before the radiation damage of the sample is manifested. ${ }^{3}$

The single-shot CDI pattern formed by ultra-short FEL pulses is also an excellent probe for transient phenomena. Analyzing a set of patterns, collected at different time delays after an excitation pulse from a pump laser in "classic" pumpprobe experiments ${ }^{4}$ or using split-delay FEL beam schemes and $\mathrm{x}$-ray photon correlation spectroscopy, ${ }^{5,6}$ the transient states of the system can be monitored on time scales determined by the FEL pulse duration only. It should be noted that, even before applying Fourier inversion methods, information

\footnotetext{
a) Present address: NSLS II, Brookhaven National Lab, P.O. Box 5000, Upton, New York 11973-5000.

b) Author to whom correspondence should be addressed. Electronic mail: kiskinova@elettra.trieste.it.
}

on the induced dynamics or changes in the average size and size distribution of the microscopic building blocks is already coded in the intensities and dimensions of CDI patterns. ${ }^{7,8}$

For CDI it is convenient when the data satisfies an oversampling constraint that requires the target to be an individual "free-standing" portion of matter: to deliver isolated objects intersecting the FEL beam hitting zone, the aerosol methodologies, that have already allowed the execution of the first single-particle CDI experiment, ${ }^{9}$ can be applied, adapted, and further developed. The aerosol science is going to play an important role in CDI experiments with FELs and, as demonstrated at FLASH,${ }^{10}$ it has already enabled some of the highest resolution imaging of single micron-sized or smaller airborne particulate matter, while preserving the native substrate-free state of the aerosol.

The wavelength tunability of FEL sources also enables resonant coherent CDI across selected atomic absorption edges, adding information about the spatial distribution of the chemical constituents of the specimen. The polarization (circular or linear) of the FEL light can extend the information to spin and orbital momentum, revealing features such as the orientation of magnetic domains, bonds in constituent molecules, etc.

The limitations for resonant CDI, imposed by the partial longitudinal coherence of the operating self-amplified stimulated emission (SASE) FELs, are going to be overcome by the nearly full coherence of the emerging seeded FEL sources, where the FEL pulses, stemming from a conventional coherent laser pulse, achieve a well-defined temporal shape 
and intensity stability. ${ }^{11}$ In principle, radiation bandwidths close to the Fourier transform limit can be obtained.

The seeded FEL Fermi@Elettra has produced the first photons and is expected to begin operation for users before the end of 2011; its seeding scheme will allow easier narrow range wavelength tunability, compared to another promising set up under development at SCSS in Harima, using highorder harmonics of a laser. ${ }^{12}$ Fermi@Elettra is opening the possibility to perform unique experiments taking advantage of the full transverse and longitudinal coherence and variable polarization of pulses with photon energies up to $300 \mathrm{eV}$ ( $900 \mathrm{eV}$ in the third harmonics). ${ }^{13}$ This will allow exploration of the structure and transient states of condensed, soft, and low-density matter by means of a great variety of diffraction, scattering, and spectroscopy techniques, implemented on three different beamlines.

In this paper we describe the multipurpose experimental station that will be operated at the coherent diffraction and projection imaging (DiProI) beamline on Fermi@Elettra. The instrument design, flexible and adaptable for modular experimental setups including exchange of sample stages, aerosol particle delivery, and detection systems, was carried out in partnership with scientists and engineers from Elettra, Lawrence Livermore National Laboratory (LLNL), the DESY Centre for Free-Electron Laser Science (CFEL), and the University of Uppsala. Commissioning of the components and performance tests have been conducted with synchrotron light at the Nanospectroscopy beamline at Elettra. ${ }^{14}$

\section{B. DiProl beamline at the Fermi@Elettra FEL}

The DiProI beamline at Fermi@Elettra will exploit the expected performance of the fully coherent seeded FEL in terms of continuum tunability, with wavelengths between 50 and $4 \mathrm{~nm}$ in the first harmonics, temporal pulse structure down to a few tens of fs at $50 \mathrm{~Hz}$ repetition rate, with $10^{13}$ photons per pulse and variable photon polarization. The beamline is designed to meet the requirements for static and dynamic CDI experiments with a resolution down to $10 \mathrm{~nm}$, while implementing options for complementary projection imaging.

Since the spectral purity of the non-monochromatized radiation beam is sufficient to perform CDI experiments, the DiProI beamline will work without any grating-based monochromator, sparing the additional efficiency loss: a limited number of individual higher harmonics will be selected, using wave-front and time-preserving multilayer mirrors, optimized to provide the maximum reflectivity at one particular wavelength, while quenching the first harmonics.

A split-delay correlation system will allow two-color experiments, splitting the beam into two parts and introducing, by means of grazing incidence optics, a delay between 0.2 and $35 \mathrm{ps}$ with a minimum step of $1 \mathrm{fs}$; larger delays, up to more than $1 \mathrm{~ns}$, will be provided by removable and interchangeable sets of four multilayer mirrors, working at $45^{\circ}$, with a total translation range of $185 \mathrm{~mm}$. The proper set of multilayers can also be chosen to suppress the fundamental wavelength and highlight the higher harmonics. ${ }^{15}$
Two custom made active mirrors, mounted in a Kirkpatrick-Baez (KB) configuration, will be used as focusing optics, crucial for imaging single small objects, providing a minimum spot size of $2.5 \times 4 \mu \mathrm{m}^{2}$ and a maximum fluence reaching $1 \times 10^{17} \mathrm{~W} / \mathrm{cm}^{2}$ at a $20 \mathrm{~nm}$ wavelength. The beam can be defocused, preserving the spot position, to provide a variable spot shape and dimension in order to illuminate larger specimen or reduce the fluence.

\section{END STATION}

\section{A. High vacuum vessel}

The DiProI end station chamber (see Fig. 1) is a parallelepiped vessel of dimensions $1100 \times 600 \times 630 \mathrm{~mm}^{3}$ composed of six $35 \mathrm{~mm}$ thick rectangular stainless steel boards; the rear panel can be replaced by an expansion module with a $320 \mathrm{~mm}$ depth to provide additional space for detectors and electronics, while one of the side panels has a rectangular 480 $\times 530 \mathrm{~mm}^{2}$ aluminum flange to provide easy access to the sample region. A deformation of less than $0.1 \mathrm{~mm}$ is expected under the operational conditions of high vacuum between 5 $\times 10^{-8}$ and $5 \times 10^{-6}$ mbar.

The vacuum is accomplished by two independent pumping systems, each composed of a $1300 \mathrm{l} / \mathrm{s}$ turbo-molecular pump, backed by a $35 \mathrm{~m}^{3} / \mathrm{h}$ scroll roughing pump, which can also be used as a prevacuum pump to lower the chamber pressure to $1 \times 10^{-3}$ mbar through an independent vacuum line. Four low vacuum valves, four high vacuum gates and the four pumps are operated by a computer system which, with the feedback of five Pirani gauges and a high vacuum pressure gauge, provides both software automated vacuum operation and programmable logic controller implemented safety reaction in case of failure.

The chamber can be vented through a nitrogen line and a manual valve; rapid changes in the setup configuration can be performed in the sample region thanks to a fast handle-locked KF160 flange. The pumping system has been dimensioned to be capable of purging aerosol samples leaking into the chamber through particle delivery systems during experiments: in a clean chamber in fixed sample mode, vacuum is restored to operational conditions within $10 \mathrm{~min}$, and reaches its best pressure in the $10^{-7}-10^{-8}$ mbar range in a few hours.

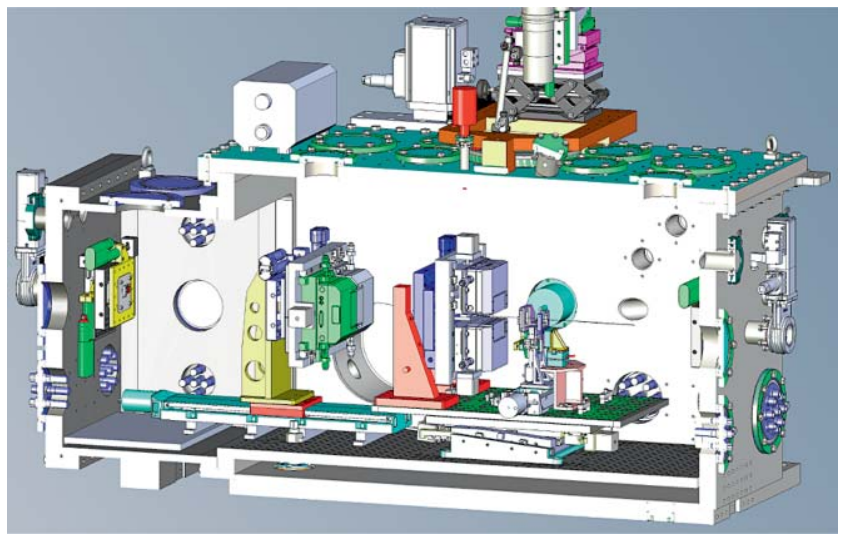

FIG. 1. (Color online): A section of the DiProI chamber design. 
Several KF40, KF100, KF160 flanges accommodate viewports, a visible light illumination source and a telescope focusing on the target surface in fixed sample mode experiments, a residual gas analyzer, electronics, and water feedthroughs; a CF100 flange on the chamber right side has been designed to accommodate an aerosol particle delivery system in correspondence with the sample-beam interaction region; five CF40 flanges host apertures converging on the sample position: two of them are used as entrance and exit windows for the FEL $\mathrm{x}$-ray beam, two are designed as laser windows at $45^{\circ}$ of incidence for time-resolved pump-probe experiments, one can house any device that has to face the sample surface, such as an evaporator or a time-of-flight mass spectrometer. The windows can be masked by black plastic covers or black rubberized fabric to minimize external light noise on the detection system.

\section{B. Light shaping and sample movement mechanics}

The chamber houses several in-vacuum computercontrolled motors to actuate the mechanics for x-ray beam shaping, cleaning and characterization, sample alignment, and observation using the optical telescope.

Entering the chamber from the beamline gate valve, the FEL pulses pass through a metal plate with cleaning apertures of different sizes, mounted on $x y$ stages actuated by stepper motors, which allow alignment to the beam. A similar set of $x y$ motorized apertures, placed before the exit gate valve at the rear side of the chamber, can host alignment and characterization devices, such as a photodiode, a wavefront sensor, etc.

All other structures (see Fig. 2) are assembled on a 950 $\times 450 \times 15 \mathrm{~mm}^{3}$ stainless steel breadboard [A], decoupled from the bottom of the chamber by a kinematic system on three contact points. The breadboard supports a set of motorized stages [B] that position a second $500 \times 270$ $\times 12 \mathrm{~mm}^{3}$ horizontal stainless steel board [C], also decoupled by a kinematic system on three contact points; in order to perform a coarse alignment with respect to the x-ray beam, the three motors of the stages can move the board with a travel range of $5 \mathrm{~mm}$ in any of the $x y z$ directions with a precision of $10 \mu \mathrm{m}$.

Before impinging on the sample, the beam is shaped, to select its coherent central part, by a circular aperture [D], positioned by two piezoelectric motors along the $x y$ directions with a precision better than $0.1 \mu \mathrm{m}$; a set of 5, 10, 20, or $50 \mu \mathrm{m}$ diameter apertures was used for tests with synchrotron radiation, reported in Sec. III. Further downstream, the beam passes through another $500 \mu \mathrm{m}$ aperture, mounted on an identical $x y$ motorized stage [E], which cleans the stray radiation before it hits the specimen, held in the x-ray beam focus.

An array of fixed targets can be mounted on a removable sample holder, hosted on a sample support [F] with four degrees of freedom: the target tip and tilt are provided by mechanics actuated by two independent picomotors. This structure is mounted on a stepper motor with a $25 \mathrm{~mm}$ travel range along $y$; a second stepper motor, with a $100 \mathrm{~mm} x$ movement,
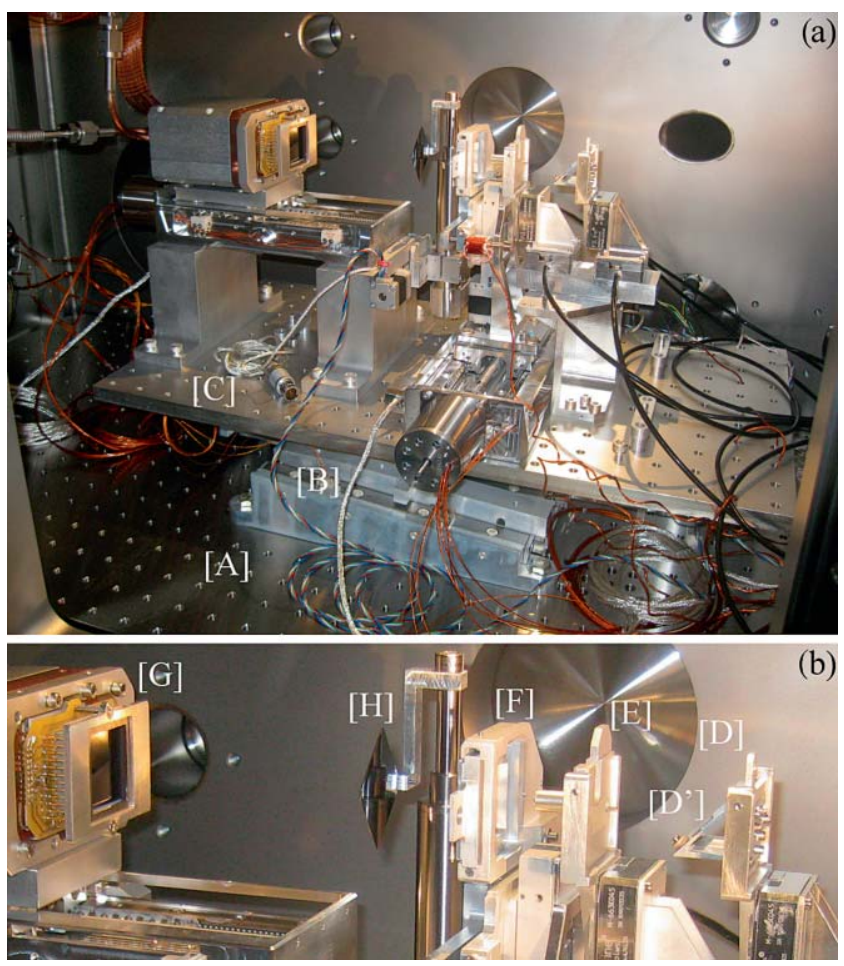

FIG. 2. (Color online): Experimental setup inside the DiProI vacuum chamber. (a) The main breadboard [A] carries the stages [B] that position the motorization board $[\mathrm{C}]$. (b) Propagating from the right, the beam passes through the apertures set [D] (carrying the $45^{\circ}$ telescope mirror [D']), the screening plate $[\mathrm{E}]$, the sample $[\mathrm{F}]$, and the beam stopper $[\mathrm{H}]$; the diffraction pattern is detected by the CCD $[\mathrm{G}]$ on the left.

carries the entire support, allowing not only control of the target's horizontal position, but also to remove the sample structure off the interaction region to safely exchange specimens. The sample support can be removed in toto to perform experiments with a particle delivery system.

The sample surface, reflected by a $45^{\circ}$ mirror [D'] mounted below the circular apertures [D], can be observed with $5 \mu \mathrm{m}$ resolution by the telescope, through a window on the chamber cover plate: in order to see the target, a $5 \mathrm{~mm} \mathrm{U}$ shaped aperture, carved off the stray radiation screening plate [E], is aligned with respect to the telescope path.

\section{Charge-coupled device (CCD) detection system}

The diffraction pattern from the sample is recorded by an $x$-ray sensitive CCD [G]. In synchrotron CDI, the central part of the detector is screened by a beam stop $[\mathrm{H}]$, since the large amount of primary unscattered photons that contribute to the pattern would damage the detector.

In the case of FEL CDI experiments, where the high fluence would damage even the beam stop, direct acquisition of the scattered patterns requires new detection systems, ${ }^{16}$ consisting of two independent CCDs separated by a variable gap, and readout time comparable with the FEL repetition rate. Since off-shelf solutions are hardly available, an indirect detection scheme $\mathrm{e}^{3,17}$ will be implemented during the commissioning phase of the DiProI beamline. 
The commissioning and first tests of the DiProI end station have been performed on the synchrotron radiation beamline Nanospectroscopy at Elettra with a Princeton Instruments PI-MTE 2048B x-ray Camera, a CCD device consisting of $2048 \times 2048$ pixels with $13.5 \times 13.5 \mu \mathrm{m}^{2}$ size; a Peltier cooler keeps the detector at $-45^{\circ} \mathrm{C}$, providing low noise operation with approximately 10 electrons RMS; the typical readout time is $4 \mathrm{~s}$ for the 4 megapixel images, with a 16 bit depth, governed by a $1 \mathrm{MHz}$ analog to digital converter.

In the classical approach with simple central beam stop protection, the detector is placed directly downstream the sample: in order to optimize the wavelength-dependent size of the acquired diffraction patterns, a $z$ motorized stage allows changing the sample-detector distance between 50 and $140 \mathrm{~mm}$ along the beam axis. A square silicon plate beam stopper $[\mathrm{H}]$ is mounted on $x y$ stages between sample and detector, keeping its diagonal along the vertical direction. The central part of the detector can be covered alternatively by the top and the bottom vertex of the beam stopper, allowing the entire diffraction pattern to be acquired in two consecutive measurements.

For the commissioning of the DiProI beamline and end station at Fermi@Elettra, where the FEL peak power prevents the use of a central beam stop, the single chip CCD detector [G] will be used in an indirect detection setup (see Fig. 3), first exploited by Chapman, ${ }^{3,17}$ to acquire the diffraction pattern reflected by a multilayer mirror [I], with a hole to let the primary beam [J] pass through and widen due to its divergence far away from the focal point before hitting a beam stopper. The mirror is mounted with a $45^{\circ}$ angle of incidence on two picomotor driven goniometers that provide tip and tilt adjustment.

Although such an indirect detection setup has been operated successfully in the past, in addition to the slow CCD readout time, it has severe limitations for exploiting the FEL tunability, since the multilayer mirrors are monochromatic, expensive, and work well only for longer wavelengths. For the commissioning with FEL light, pilot experiments will

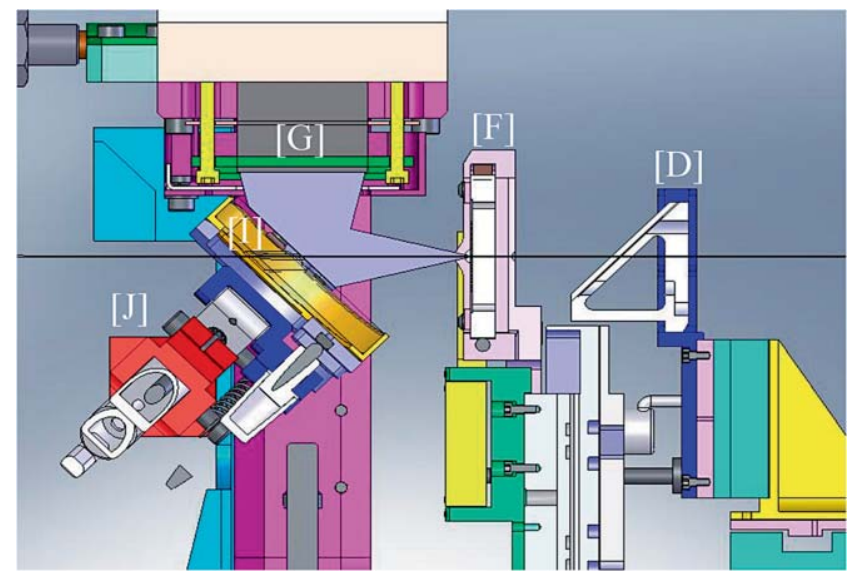

FIG. 3. (Color online): Design of the DiProI indirect acquisition set up using a multilayer deflecting mirror. Propagating from the right, the beam passes through the apertures stage $[\mathrm{D}]$, the sample $[\mathrm{F}]$, and the multilayer mirror $[\mathrm{I}]$, which deflects the diffraction image to the $\mathrm{CCD}[\mathrm{G}]$, letting the primary beam [J] through a hole.

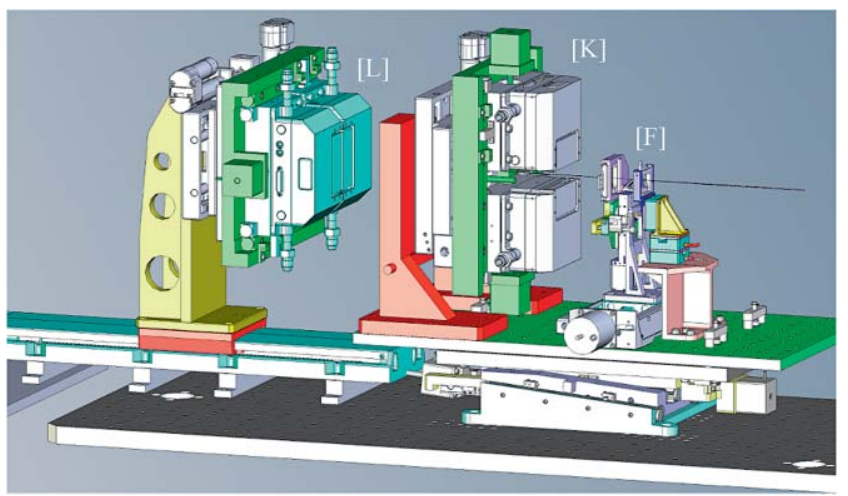

FIG. 4. (Color online): The most desired direct acquisition set-up using two detector systems (to be implemented). Photons scattered from the sample $[\mathrm{F}]$ are detected by a near $[\mathrm{K}]$ or a far $[\mathrm{L}] \mathrm{CCD}$ system, depending on the deflection angle.

be performed with the currently available $32,16,13.5$, and $10 \mathrm{~nm}$ mirrors. However, in parallel, possible solutions are under consideration to implement direct detection before the user operation state, to allow a large variety of CDI experiments, including resonant CDI and time-resolved studies.

The envisaged final configuration of a direct acquisition setup (see Fig. 4), as successfully implemented in the MPICAMP chamber using pn-CCDs, ${ }^{16}$ combines two fast readout detectors $[\mathrm{K}]$ close to the sample with two similar modules [L] placed further down the beam path, to monitor simultaneously both wide and small scattering angles.

\section{COMMISSIONING AND FIRST TESTS WITH SYNCHROTRON LIGHT}

\section{A. Experimental setup commissioning}

The DiProI end station's vacuum chamber, designed, built, and assembled between 2008 and 2010, has undergone commissioning tests of its experimental setup at the nanospectroscopy beamline at Elettra that provides a synchrotron radiation beam with multiple polarization, moderately focused by a KB mirror system to a $150 \times 100 \mu \mathrm{m}^{2}$ spot size. ${ }^{14}$ The CDI patterns are detected with the CCD-central stop configuration described in Secs. II A and II B.

\section{B. Two pin hole Young experiment interference test}

The first tests were dedicated to characterize the transverse coherence of the focused synchrotron beam: horizontal coherence was measured performing the classic Young experiment on a set of samples comprising double pin holes with a pitch ranging from 0.5 to $11 \mu \mathrm{m}$; to determine the vertical coherence, double slit targets with the same separation range were used. Design parameters of the samples are reported in Table I.

The structures are realized using focused ion beam (FIB) milling of the $1 \mu \mathrm{m} \mathrm{W}$ film deposited by sputtering on $\mathrm{Si}_{3} \mathrm{~N}_{4}$ windows, $30 \mathrm{~nm}$ thick and $100 \mu \mathrm{m}$ wide, etched on a Si wafer. The X-ray source, with photon energy set to 250,500 , or $700 \mathrm{eV}$, was defined by a $20 \mu \mathrm{m}$ diameter aperture, placed at a distance of $90 \mathrm{~mm}$ in front of the sample. 
TABLE I. Design parameters of the samples used to test the beam transversal coherence.

\begin{tabular}{|c|c|c|c|c|}
\hline \multicolumn{2}{|c|}{ Horizontal coherence } & \multicolumn{3}{|c|}{ Vertical coherence } \\
\hline Pin hole separation & Pin hole diameter & Slit separation & Slit width & Slit height \\
\hline $0.5 \mu \mathrm{m}$ & $170 \mathrm{~nm}$ & $0.5 \mu \mathrm{m}$ & $170 \mathrm{~nm}$ & $700 \mathrm{~nm}$ \\
\hline $1.5 \mu \mathrm{m}$ & $170 \mathrm{~nm}$ & $1.5 \mu \mathrm{m}$ & $170 \mathrm{~nm}$ & $700 \mathrm{~nm}$ \\
\hline $3 \mu \mathrm{m}$ & $170 \mathrm{~nm}$ & $3 \mu \mathrm{m}$ & $170 \mathrm{~nm}$ & $700 \mathrm{~nm}$ \\
\hline $5 \mu \mathrm{m}$ & $180 \mathrm{~nm}$ & $5 \mu \mathrm{m}$ & $170 \mathrm{~nm}$ & $700 \mathrm{~nm}$ \\
\hline $7 \mu \mathrm{m}$ & $180 \mathrm{~nm}$ & $\ldots$ & $\ldots$ & $\ldots$ \\
\hline $11 \mu \mathrm{m}$ & $210 \mathrm{~nm}$ & $11 \mu \mathrm{m}$ & $200 \mathrm{~nm}$ & $800 \mathrm{~nm}$ \\
\hline
\end{tabular}

A commonly accepted ${ }^{18}$ parameter to define the degree of coherence of a monochromatic light source, with wavelength $\lambda$ and dimension radius $R$, is the visibility $V=\left(I_{\max }\right.$ $\left.-I_{\min }\right) /\left(I_{\max }+I_{\min }\right)$, where $I_{\max }$ and $I_{\min }$ are the maximum and minimum of the interference fringes of two pin holes with separation $\zeta$, placed at a distance $L$ from the source. The theoretical prediction is given by $V=\left|2 J_{1}(x) / x\right|^{2}$, where $J_{1}(x)$ is the Bessel function of first kind and $x=2 \pi R \zeta / \lambda L$.

The selected results of the coherence tests shown in Fig. 5 are obtained with $250 \mathrm{eV}$ photon energy and a target to
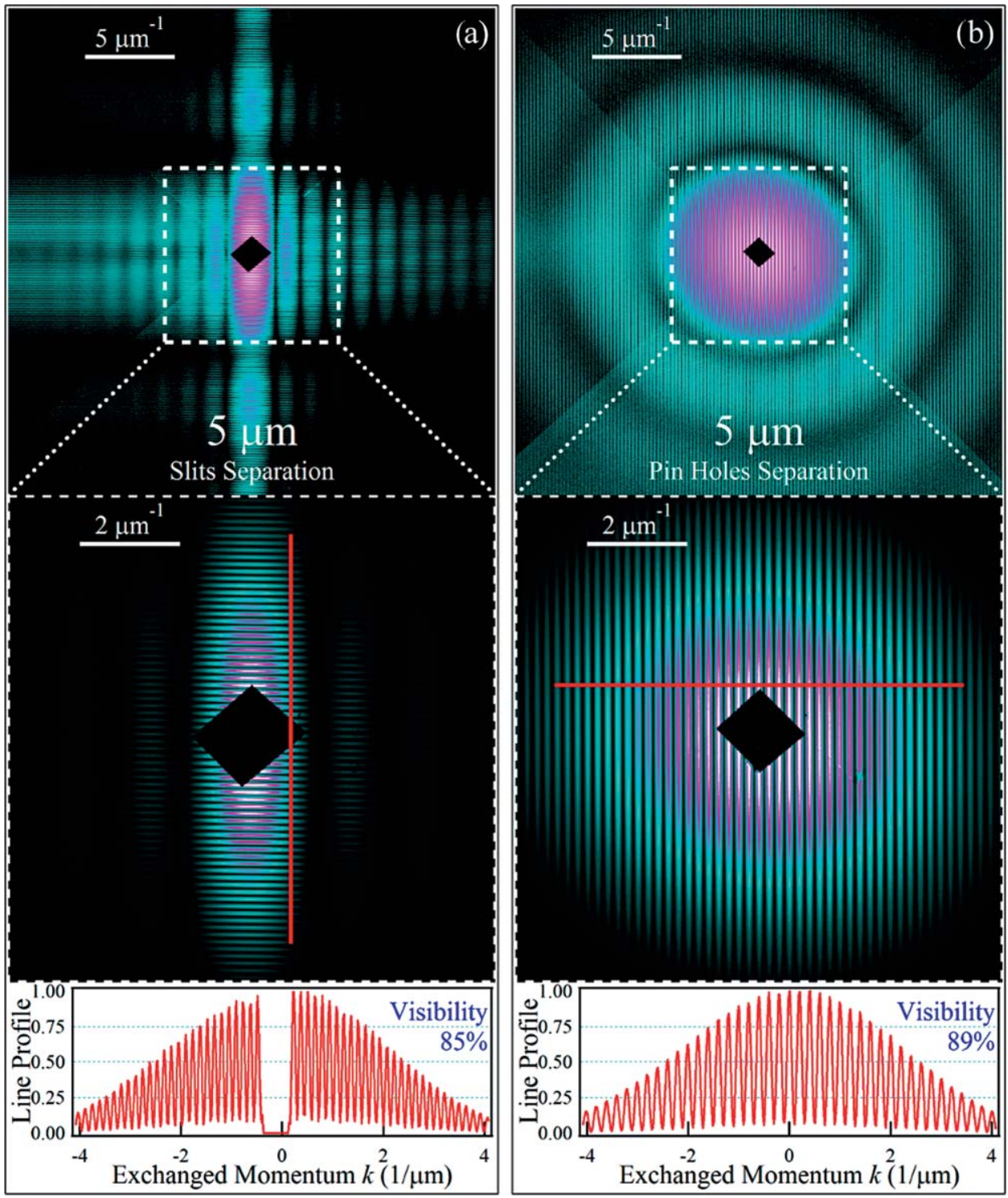

FIG. 5. (Color online): Coherence test examples taken at a photon energy of $250 \mathrm{eV}$. (a) Double slits with $5 \mu \mathrm{m}$ vertical separation. (b)-(d) Double pin holes with horizontal separation 5, 3, and $0.5 \mu \mathrm{m}$, respectively. Top panels show the diffraction patterns (log scale), middle panels outline the interference fringes (linear scale) and bottom panels show the intensity modulation profiles taken along the red lines in the images. (e) Fringes visibility data compared to the theoretical predictions as a function of pin holes or slits separation, for different photon energies. 

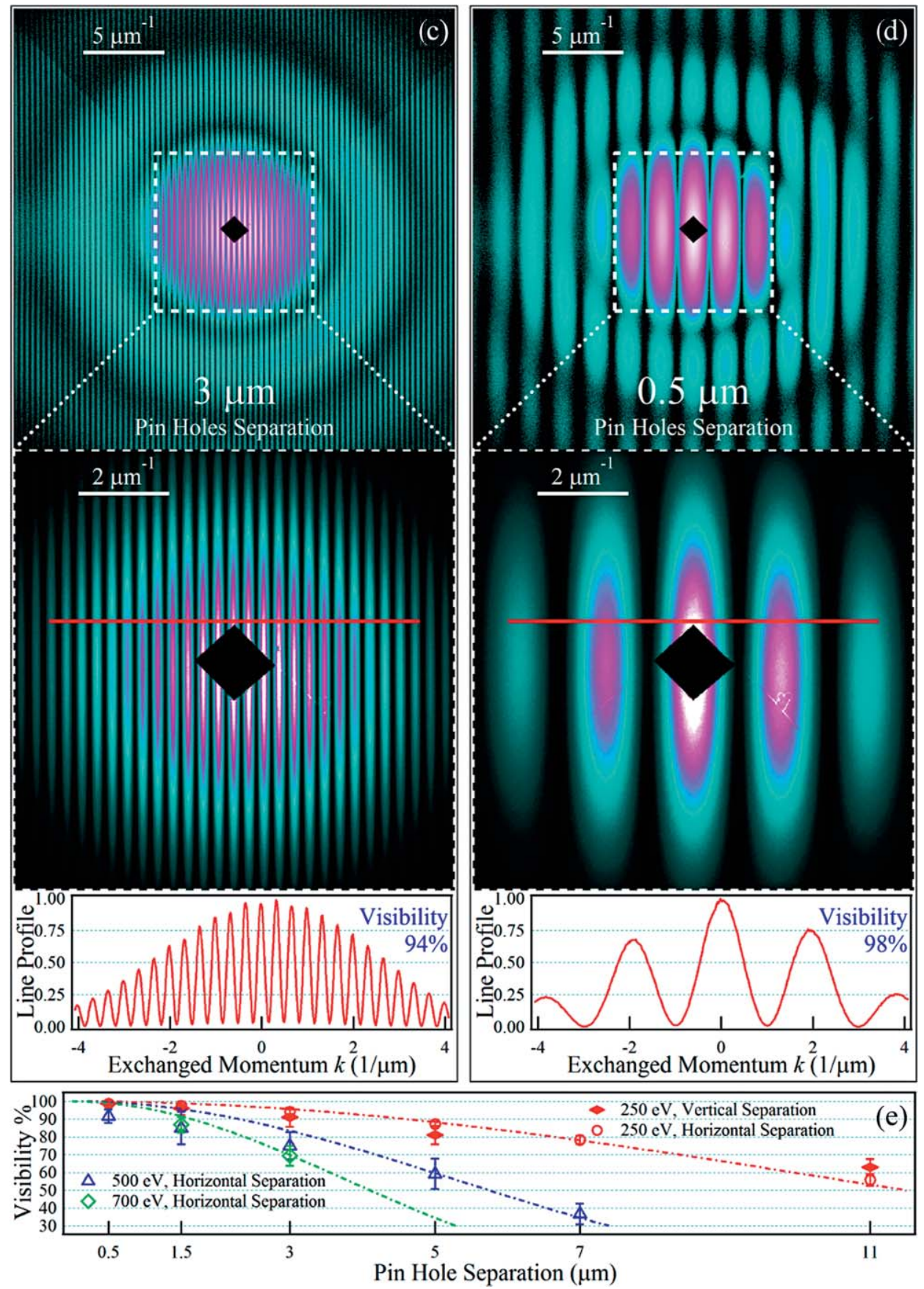

FIG. 5. (Continued)
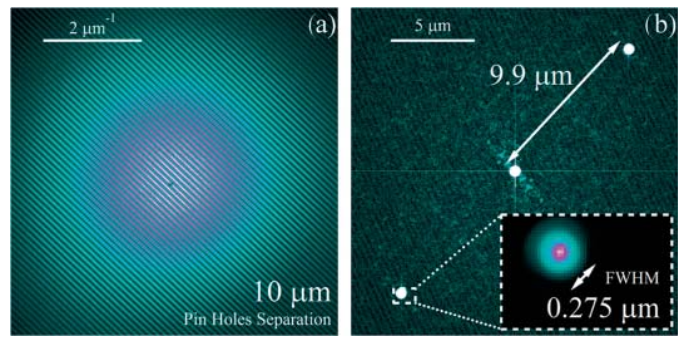

FIG. 6. (Color online): Data obtained with $250 \mathrm{eV}$ x-rays from two $300 \mathrm{~nm}$ pin holes with a $10 \mu \mathrm{m}$ diagonal separation. (a) Central part of the diffraction pattern. (b) Fourier transform of the double pinhole diffraction pattern: the holes autocorrelation is in the image center; their holographic images (one is magnified in the box) stand in symmetric positions. detector distance of $140 \mathrm{~mm}$. The images are obtained with a $5 \mu \mathrm{m}$ vertically separated double slit [Fig. 5(a)], and 5,3 , and $0.5 \mu \mathrm{m}$ horizontally separated double pin holes [Fig. 5(b)-5(d)].

The top $1400 \times 1400$ pixels images show the diffraction pattern for a collecting angle of $\pm 3.9^{\circ}$, corresponding to a maximum exchange momentum transfer of $\pm 13.6 \mu \mathrm{m}^{-1}$; the logarithmic scale, enhancing low intensity features, allows distinguishing several orders of the slit diffraction envelope [Fig. 5(a)] and of the pin hole Airy pattern [Fig. 5(b)-5(d)]. The dark areas in the middle of the diffraction patterns is due to the beam stop protecting the CCD chip from the direct $\mathrm{x}$-ray beam: the images are obtained combining two 
acquisitions with different beam stop positions. The expanded central part of the images show in linear scale the interference fringes, whose visibility is illustrated by the intensity modulation profiles in the bottom panels.

The results of the coherence tests are summarized in Fig. 5(e), where the experimental visibility values (markers), obtained for several samples at different wavelengths, is compared to the theoretical predictions (dash dotted lines). For $250 \mathrm{eV}$ photons, the horizontal and vertical transverse coherence are comparable, with a fringe visibility in the range spanning from $99 \pm 1 \%$ to $56 \pm 3 \%$ while the objects separation varies between 0.5 and $11 \mu \mathrm{m}$. For higher photon energies, the fringe visibility decreases with the increase of the adimensional parameter $x$, remaining however greater than $50 \%$ for an object separation smaller than $4 \mu \mathrm{m}$.

In order to evaluate the effect of a partially coherent beam (i.e., a fringe visibility of about $60 \%$ ) on the imaging properties of the experimental setup, an ad hoc sample with two $300 \mathrm{~nm}$ pin holes with a $10 \mu \mathrm{m}$ diagonal separation, was illuminated with $250 \mathrm{eV}$ photons through a $50 \mu \mathrm{m}$ diameter cleaning aperture.

Figure 6(a) shows the interference fringes in the central part of the diffraction pattern, while Fig. 6(b) presents the Fourier transform of the acquired interference pattern: despite the partial coherence of the beam, three sharp and intense spots are clearly visible in the centrosymmetric image. The central dot is the autocorrelation function of the two pin holes; the two lateral spots are their holographic images, with $275 \pm 10 \mathrm{~nm}$ FWHM and $9.91 \pm 0.01 \mu \mathrm{m}$ distance from the center. These values are close to the nominal sample specifications, showing that the technique is quite robust to provide high resolution images, even under partially coherent illumination.

\section{Resonant CDI test across the Co $L_{3}$ edge of nanostructured specimen}

One of the peculiar features of photons with respect to electrons is longer penetration depth, making photon based techniques more appealing to detect and image materials buried within a solid, without destroying the sample.

The advantage of using $\mathrm{x}$ rays with wavelengths tuned to the atomic absorption resonances to perform resonant CDI is that the abrupt change of the optical absorption and refractive factors across the atomic edges adds information about the elemental lateral distribution in complex nanostructured specimens. ${ }^{19,20}$ The non-negligible differences between the speckle patterns, taken at and away of a selected atomic $K$, $L$, or $M$ edge resonance, not only provide elemental information but are also sensitive to dichroic effects.

The test experiments demonstrating the applicability of resonant CDI for elemental and magnetic imaging are performed with a specimen of Co square islands, fabricated by FIB on a $\mathrm{Si}_{3} \mathrm{~N}_{4}$ substrate.

Figure 7 compares the diffraction patterns obtained with photon energies of 770, 778, and $785 \mathrm{eV}$, i.e., below, at, and above the $\mathrm{Co}_{3}$ edge, respectively; the images are scaled to a square root to enhance low intensity features. It is clearly visible that the speckle pattern changes at the resonance energy: the long and narrow spots, observed in the diffraction pattern below the cobalt adsorption edge [Fig. 7(a)], lose intensity due to the onset of the isotropic resonance scattering [Fig. 7(b)], while, moving further above the edge, the pattern partially recovers the features observed below the resonance energy [Fig. 7(c)].

The ferromagnetic cobalt sample also allowed a sensitivity test for magnetic imaging, ${ }^{21,22}$ using right and left

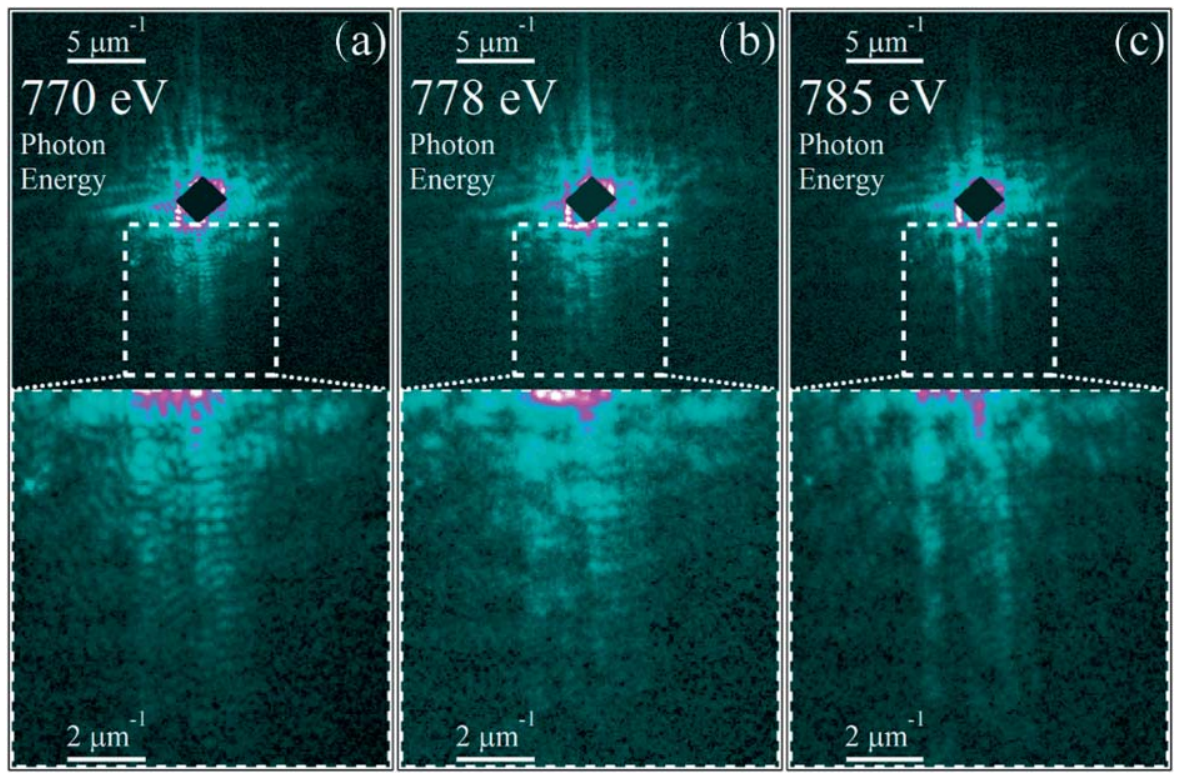

FIG. 7. (Color online): Diffraction patterns obtained from a cobalt sample at different photon energies (square root scale). (a) 770 eV, below the Co $\mathrm{L}_{3}$ edge. (b) $778 \mathrm{eV}$, resonant with the $\mathrm{Co} \mathrm{L}_{3}$ edge. (c) $785 \mathrm{eV}$, above the $\mathrm{Co} \mathrm{L}_{3}$ edge. The fluctuations in the intensity of the features at and away of the resonance smaller areas are well visible in the enlarged images of the selected section shown in the lower panels. 

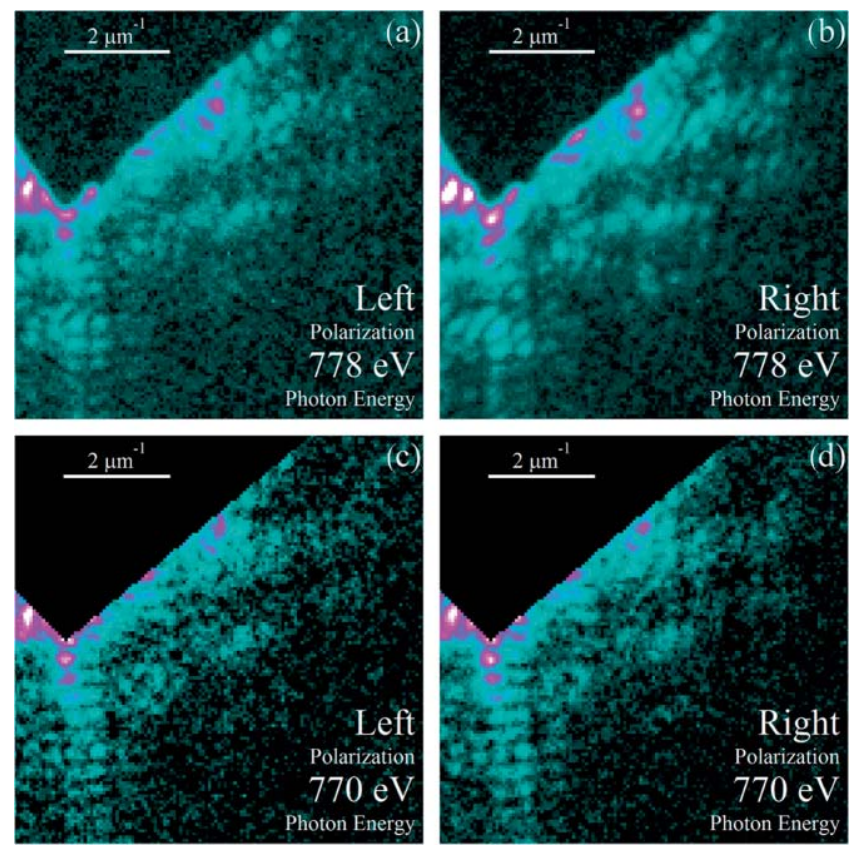

FIG. 8. (Color online): Enlarged areas of diffraction patterns from a cobalt sample using circularly polarized light (square root scale). (a) and (b) Dichroism is observed changing light polarization chirality at $778 \mathrm{eV}$ photon energy, resonant with the $\mathrm{Co} L_{3}$ edge. (c) and (d) At $770 \mathrm{eV}$ photon energy, below the Co $L_{3}$ edge resonance, no relevant difference is found changing polarization chirality.

circularly polarized light: Fig. 8 shows, in square root intensity scale, areas of the diffraction patterns taken at and below the $\mathrm{Co}_{3}$ resonance using right [Figs. 8(a), 8(c)] and left [Figs. 8(b), 8(d)] circular polarization.

Comparing Figs. 8(a) and 8(b), taken at the Co $L_{3}$ edge $(778 \mathrm{eV})$ using beam with opposite chirality, significant differences, due to the dichroic effects, can be observed. On the contrary, no evident difference can be found comparing the patterns in Figs. 8(c) and 8(d), taken with photon energy below the $\mathrm{Co} L_{3}$ edge $(770 \mathrm{eV})$.

\section{CONCLUSION AND OUTLOOK}

The flexible end station for the DiProI beamline at Fermi@Elettra has been designed, assembled, and has undergone the commissioning tests using partially coherent synchrotron light. The results from the first test experiments proved that all components perform according to their specifications. This makes us confident that, after commissioning with FEL beam in the first months of 2011, the modular DiProI station, planned for a great variety of CDI experiments, will welcome the first users.

Among the important applications of the DiProI station at the soft x-ray Fermi facility, can be single-shot fs coherent diffractive imaging of living cells. Indeed, since the Fermi wavelengths are limited to $4 \mathrm{~nm}$ in the first harmonics, we cannot reach an atomic resolution, but in fact understanding many physiological functions of the living cells requires morphological information with resolution of tens $\mathrm{nm}$ for monitoring the unique conformation states of the constituents of these dynamic systems. The feasibility is demonstrated by the first pioneering experiments performed at FLASH using 13.6 and $8 \mathrm{~nm}$ wavelengths. ${ }^{23,24}$

As already discussed in Ref. 25, the most adequate pulse wavelength range for obtaining good CDI contrast of cells with sufficient penetration depth lies between the $\mathrm{C} 1 s$ and $\mathrm{O} 1 s$ edge ( 4.8 to $2.5 \mathrm{~nm}$ ). Another advantage demonstrated by the calculations is that, since the deposited energy is smaller compared to the case using short wavelengths, the radiation damage is still negligible for pulse lengths even longer than $50 \mathrm{fs}$. For example, the recent fast growing use of nanoparticles for biomedical applications has imposed urgent request for understanding the cellular responses. In this respect, observing the effect of different agents on morphology modifications of single-cell organism with resolution better than $10 \mathrm{~nm}$ is of crucial importance. The agents can penetrate different cell organelles: their presence, as well as the relevant changes in the local or entire morphology of the cells, will be reflected in the speckle pattern. The delivery of "free-standing" cells to the FEL interaction zone is an almost solved problem, thanks to the fast developing aerosol methodologies that have already demonstrated their potentials, in execution of the first single-particle CXDI experiments, ${ }^{9}$ and are planned to be implemented in the DiProI station.

The DiProI station can also be used to explore the behavior of anisotropic nanostructures, fabricated by different elements, under "extreme conditions." The experiments will monitor the "structural" evolution of the constituent elements at different time scales, using as a pump an optical laser or a split in time FEL pulse. Such studies will be of particular interest when the anisotropic targets are fabricated from materials with different melting temperature and electronic structure, so that reaching nonequilibrium conditions is selective for each structure. Using intense short FEL pulses with proper energy above the electronic edge of one of the elements, highly ionized transparent states can be created, evolving into uniformly heated warm dense matter, as demonstrated at FLASH for Al samples. ${ }^{26}$ For anisotropic targets, the set of speckles taken at different delay times after irradiation with short pulses at wavelengths matching the edges of one of the constituent elemental structure, will provide unique information about the local variation of electron densities and spatial elemental distribution resulting from nonthermal melting.

The improved parameters of the pulses generated using the seeding scheme at Fermi, in particular the full longitudinal coherence, is opening new opportunities for resonant coherent imaging. One important application field is exploring the multiple polarization of the Fermi pulses through resonant $\mathrm{CDI}$, used to enhance the magnetization scattering contrast by tuning the pulse wavelengths to the $M$ (1st harmonics) or $L$ (higher harmonics) edges of the relevant elements. The feasibility of these experiments has already been demonstrated at FLASH, using the 1st and 5th harmonics, where even the effect of the different energy deposited due to the jitter in the pulse intensity on the magnetic domain sizes was reflected in the CDI images. ${ }^{27}$

Thanks to the high number of fully coherent photons in the less than $50 \mathrm{fs}$ single Fermi pulse, 3-6 orders of 
magnitude superior than those provided at synchrotron facilities, both static and dynamic studies are possible in a singleshot mode. In particular, as unique potential, we consider using the highly focused Fermi pulses for tracking the sub-ps temporal response of magnetization density and domain dynamics in nanostructures triggered with an optical laser pump. The feasibility of another set of experiments, implementing an autocorrelator to split the incident beam not only in time, but also in space, is also under consideration at Fermi. This will allow probing the dynamics in two different specimen areas, as has been reported very recently. ${ }^{28}$

\section{ACKNOWLEDGMENTS}

The construction of the apparatus was funded by the Fermi@Elettra project. This work was in part supported by the U.S. Department of Energy (DOE), Office of Basic Energy Sciences through the PULSE Institute at the SLAC National Accelerator Laboratory, and Department of Energy's National Nuclear Security Administration, Lawrence Livermore National Security.

${ }^{1}$ J. Kirz, Nat. Phys. 2, 799 (2006).

${ }^{2}$ K. J. Gaffney and H. N. Chapman, Science 316, 1444 (2007).

${ }^{3}$ H. N. Chapman, A. Barty, M. J. Bogan, S. Boutet, M. Frank, S. P. Hau-Riege, S. Marchesini, B. W. Woods, S. Bajt, W. H. Benner, R. A. London, E. Plönjes, M. Kuhlmann, R. Treusch, S. Düsterer, T. Tschentscher, J. R. Schneider, E. Spiller, T. Möller, C. Bostedt, M. Hoener, D. A. Shapiro, K. O. Hodgson, D. Van Der Spoel, F. Burmeister, M. Bergh, C. Caleman, G. Huldt, M. M. Seibert, F. R. N. C. Maia, R. W. Lee, A. Szöke, N. Timneanu, and J. Hajdu, Nat. Phys. 2, 839 (2006).

${ }^{4}$ A. Barty, S. Boutet, M. J. Bogan, S. Hau-Riege, S. Marchesini, K. Sokolowski-Tinten, N. Stojanovic, R. Tobey, H. Ehrke, A. Cavalleri, S. Düsterer, M. Frank, S. Bajt, B. W. Woods, M. M. Seibert, J. Hajdu, R. Treusch, and H. N. Chapman, Nat. Photonics 2, 415 (2008).

${ }^{5}$ H. N. Chapman, S. P. Hau-Riege, M. J. Bogan, S. Bajt, A. Barty, S. Boutet, S. Marchesini, M. Frank, B. W. Woods, W. H. Benner, R. A. London, U. Rohner, A. Szöke, E. Spiller, T. Möller, C. Bostedt, D. A. Shapiro, M. Kuhlmann, R. Treusch, E. Plönjes, F. Burmeister, M. Bergh, C. Caleman, Gösta Huldt, M. M. Seibert, and J. Hajdu, Nature (London) 448, 676 (2007).

${ }^{6}$ G. Grübel, G. B. Stephenson, C. Gutt, H. Sinn, and T. Tschentscher, Nucl. Instrum. Methods Phys. Res. B 262, 357 (2007).

${ }^{7}$ F. Van Der Veen and F. Pfeiffer, J. Phys.: Condens. Matter. 16, 5003 (2004).

${ }^{8}$ K. A. Nugent, Adv. Phys. 59, 1 (2010).

${ }^{9}$ M. J. Bogan, W. H. Benner, S. Boutet, U. Rohner, M. Frank, A. Barty, M. M. Seibert, F. Maia, S. Marchesini, S. Bajt, B. Woods, V. Riot, S. P. Hau-Riege, M. Svenda, E. Marklund, E. Spiller, J. Hajdu, and H. N. Chapman, Nano Lett. 8, 310 (2008).

${ }^{10}$ M. J. Bogan, D. Starodub, C. Y. Hampton, and R. G. Sierra, J. Phys. B 43, 194013 (2010).

${ }^{11}$ L. H. Yu, L. DiMauro, A. Doyuran, W. S. Graves, E. D. Johnson, R. Heese, S. Krinsky, H. Loos, J. B. Murphy, G. Rakowsky, J. Rose, T. Shaftan, B. Sheehy, J. Skaritka, X. J. Wang, and Z. Wu, Phys. Rev. Lett. 91, 074801 (2003).

${ }^{12}$ T. Togashi, E. J. Takahashi, K. Midorikawa, M. Aoyama, K. Yamakawa, T. Sato, A. Iwasaki, S. Owada, T. Okino, K. Yamanouchi, F. Kannari, A. Yagishita, H. Nakano, M. E. Couprie, K. Fukami, T. Hatsui, T. Hara, T. Kameshima, H. Kitamura, N. Kumagai, S. Matsubara, M. Nagasono, H. Ohashi, T. Ohshima, Y. Otake, T. Shintake, K. Tamasaku, H. Tanaka,
T. Tanaka, K. Togawa, H. Tomizawa, T. Watanabe, M. Yabashi, and T. Ishikawa, Opt. Express 19, 317 (2010).

${ }^{13}$ E. M. Allaria, C. Callegari, D. Cocco, W. M. Fawley, M. Kiskinova, C. Masciovecchio, and F. Parmigiani, New J. Phys. 12, 075002 (2010).

${ }^{14}$ A. Locatelli, A. Bianco, D. Cocco, S. Cherifi, S. Heun, M. Marsi, M. Pasqualetto, and E. Bauer, J. Phys. IV France 104, 99 (2003).

${ }^{15}$ M. G. Pelizzo, A. J. Corso, G. Monaco, P. Nicolosi, M. Suman, P. Zuppella, and D. Cocco, Nucl. Instrum. Methods Phys. Res. A 635, S24 (2011).

${ }^{16}$ L. Strüder, S. Epp, D. Rolles, R. Hartmann, P. Holl, G. Lutz, H. Soltau, R. Eckart, C. Reich, K. Heinzinger, C. Thamm, A. Rudenko, F. Krasniqi, K.-U. Kühnel, C. Bauer, C.-D. Schröter, R. Moshammer, S. Techert, D. Miessner, M. Porro, O. Hälker, N. Meidinger, N. Kimmel, R. Andritschke, F. Schopper, G. Weidenspointner, A. Ziegler, D. Pietschner, S. Herrmann, U. Pietsch, A. Walenta, W. Leitenberger, C. Bostedt, Möller, D. Rupp, M. Adolph, H. Graafsma, H. Hirsemann, K. Gärtner, R. Richter, L. Foucar, R. L. Shoeman, I. Schlichting, and J. Ullrich, Nucl. Instrum. Methods Phys. Res. A 614, 483 (2010).

${ }^{17}$ S. Bajt, H. N. Chapman, E. A. Spiller, J. B. Alameda, B. W. Woods, M. Frank, M. J. Bogan, A. Barty, S. Boutet, S. Marchesini, S. P. Hau-Riege, J. Hajdu, and D. Shapiro, Appl. Opt. 47, 1673 (2008).

${ }^{18}$ G. O. Reynolds, J. B. DeVelis, G. B. Parrent, Jr., and B. J. Thompson, The New Physical Optics Notebook: Tutorials in Fourier Optics, s.1. (SPIE Optical Engineering Press, Bellingham, Washington, 1989).

${ }^{19}$ C. Song, R. Bergstrom, D. Ramunno-Johnson, H. Jiang, D. Paterson, M. D. de Jonge, I. McNulty, J. Lee, K. L. Wang, and J. Miao, Phys. Rev. Lett. 100, 025504 (2008).

${ }^{20}$ Y. Takahashi, H. Kubo, H. Furukawa, K. Yamauchi, E. Matsubara, T. Ishikawa, and Y. Nishino, Phys. Rev. B 78, 092105 (2008).

${ }^{21}$ S. Eisebitt, J. Lüning, W. F. Schlotter, M. Lörgen, O. Hellwig, W. Eberhardt, and J. Stöhr, Nature (London) 432, 885 (2004).

${ }^{22}$ C. Gutt, L.-M. Stadler, S. Streit-Nierobisch, A. P. Mancuso, A. Schropp, B. Pfau, C. M. Günther, R. Könnecke, J. Gulden, B. Reime, J. Feldhaus, E. Weckert, I. A. Vartanyants, O. Hellwig, F. Staier, R. Barth, M. Grunze, A. Rosenhahn, D. Stickler, H. Stillrich, R. Frömter, H. P. Oepen, M. Martins, T. Nisius, T. Wilhein, B. Faatz, N. Guerassimova, K. Honkavaara, V. Kocharyan, R. Treusch, E. Saldin, S. Schreiber, E. A. Schneidmiller, M. V. Yurkov, S. Eisebitt, and G. Grübel, Phys. Rev. B 79, 212406 (2009).

${ }^{23}$ M. M. Seibert, S. Boutet, M. Svenda, T. Ekeberg, F. R. N. C. Maia, M. J. Bogan, N. Tîmneanu, A. Barty, S. Hau-Riege, C. Caleman, M. Frank, H. Benner, J. Y. Lee, S. Marchesini, J. W. Shaevitz, D. A. Fletcher, S. Bajt, I. Andersson, H. N. Chapman, and J. Hajdu, J. Phys. B 43, 194015 (2010).

${ }^{24}$ A. P. Mancuso, T. Gorniak, F. Staier, O. M. Yefanov, R. Barth, C. Christophis, B. Reime, J. Gulden, A. Singer, M. E. Pettit, T. Nisius, T. Wilhein, C. Gutt, G. Grübel, N. Guerassimova, R. Treusch, J. Feldhaus, S. Eisebitt, E. Weckert, M. Grunze, A. Rosenhahn, and I. A. Vartanyants, New J. Phys. 12, 035003 (2010).

${ }^{25}$ M. Bergh, N. Tîmneanu, G. Huldt, H. A. Scott, and J. Hajdu, Q. Rev. Biophys. 41, 181 (2008).

${ }^{26}$ B. Nagler, U. Zastrau, R. R. Fäustlin, S. M. Vinko, T. Whitcher, A. J. Nelson, R. Sobierajski, J. Krzywinski, J. Chalupsky, E. Abreu, S. Bajt, T. Bornath, T. Burian, H. N. Chapman, J. Cihelka, T. Döppner, S. Düsterer, T. Dzelzainis, M. Fajardo, E. Förster, C. Fortmann, E. Galtier, S. H. Glenzer, S. Göde, G. Gregori, V. Hajkova, P. Heimann, L. Juha, M. Jurek, F. Y. Khattak, A. R. Khorsand, D. Klinger, M. Kozlova, T. Laarmann, H. J. Lee, R. W. Lee, K.-H. Meiwes-Broer, P. Mercere, W. J. Murphy, A. Przystawik, R. Redmer, H. Reinholz, D. Riley, G. Röpke, F. Rosmej, K. Saksl, R. Schott, R. Thiele, J. Tiggesbäumker, S. Toleikis, T. Tschentscher, I. Uschmann, H. J. Vollmer, and J. Wark, Nat. Phys. 5, 693 (2009).

${ }^{27}$ C. Gutt, S. Streit-Nierobisch, L.-M. Stadler, B. Pfau, C. M. Günther, R. Könnecke, R. Frömter, A. Kobs, D. Stickler, H. P. Oepen, R. R. Fäustlin, R. Treusch, J. Feldhaus, E. Weckert, I. A. Vartanyants, M. Grunze, A. Rosenhahn, T. Wilhein, S. Eisebitt, and G. Grübel. Phys. Rev. B 81, 100401(R) (2010).

${ }^{28}$ C. M. Gunther, B. Pfau, R. Mitzner, B. Siemer, S. Roling, H. Zacharias, O. Kutz, I. Rudolph, D. Schondelmaier, R. Treusch, and S. Eisebitt, Nat. Photonic 5, 99 (2011). 